\title{
Construcción del concepto de integral definida usando geometría dinámica utilizando distintos sistemas de representación
}

\author{
María Carmen Aranda López ${ }^{1}$ \\ carmen.arlo@gmail.com \\ https://orcid.org/0000-0001-5966-8599 \\ María Luz Callejo de La Vega ${ }^{2}$ \\ luz.callejo@ua.es \\ http://orcid.org/0000-0002-2617-9657 \\ ${ }^{1}$ IES Marina Baixa Generalitat Valenciana (España) \\ ${ }^{2}$ Universidad de Alicante (UA, España)
}

Recibido: 29/05/2020 Aceptado: 29/07/2020

\section{Resumen}

El objetivo de este artículo es (i) presentar tres tareas propuestas a estudiantes de 17-18 años, que se apoyan en applets de Geometría dinámica y en una hoja de cálculo, para trabajar el concepto de integral definida como límite y que se enmarcan en una unidad didáctica sobre la integral definida, e (ii) identificar distintos perfiles de estudiantes tras la realización de éstas. En las tareas se hace hincapié en la construcción de las aproximaciones dinámica y métrica del límite y la coordinación de ambas, así como en la coordinación entre distintos modos de representación. Las tareas se realizaron por parejas para favorecer la interacción y la discusión. Los resultados muestran distintos perfiles con relación a la construcción de la integral como límite y al significado de las sumas de Darboux y al error de la aproximación. Estos perfiles difieren en cuanto al grado de construcción del concepto de integral definida.

Palabras clave: Applets; Geometría dinámica; Integral definida; Aproximación al área; Sistemas de representación.

\section{Construction of the concept of defined integral using dynamic geometry and different systems of representation}

\begin{abstract}
The aim of this article is (i) to present three tasks proposed to 17-18 year-old students, which are based on dynamic geometry applets and a spreadsheet, to work on the concept of the definite integral as a limit and which are framed in a didactic unit on the defined integral, and (ii) to identify different student profiles after the completion of the tasks. In the tasks, emphasis is placed on the construction of the dynamic and metric approximations of the limit and the coordination of both, as well as on the coordination between different modes of representation. The tasks were done in pairs to encourage interaction and discussion. The results show different profiles in relation to the construction of the integral as a limit and the meaning of Darboux sums and the error of the approximation.
\end{abstract}

Keywords: Applets; Dynamic geometry; Definite integral; Approach to the area; Systems of representation. 


\section{Construção do conceito de integral definida usando geometria dinâmica usando diferentes sistemas de representação}

\section{Resumo}

O objetivo do artigo é (i) apresentar três tarefas propostas a alunos de 17 a 18 anos, apoiadas em applets de geometria dinâmica e planilha, para abordar o conceito de integral definida como limite e que se enquadram em uma unidade didática sobre a integral definida; e (ii) identificar diferentes perfis de alunos após concluí-los. As tarefas enfatizam a construção das aproximações dinâmicas e métricas do limite e a coordenação de ambas as aproximações, bem como a coordenação entre diferentes formas de representação. As tarefas foram resolvidas em duplas para favorecer a interação e a discussão. Os resultados mostram diferentes perfis em relação à construção da integral como limite e ao significado das somas de Darboux e do erro de aproximação. Esses perfis diferem no grau de construção do conceito integral definido.

Palavras-chave: Applets; Geometria dinâmica; Integral definida; Aproximação da área; Sistemas de representação.

\section{Introducción}

El estudio del Cálculo presenta dificultades a los estudiantes porque se basa en la comprensión de conceptos vinculados a procesos infinitos. Su enseñanza muchas veces se focaliza en aspectos procedimentales (Azcárate et al., 1996; González-Martín \& Camacho, 2004) y no conceptuales. Para fomentar la comprensión de estos conceptos, y específicamente el de integral, las investigaciones destacan la importancia de usar múltiples representaciones (geométricas, numéricas y algebraicas), así como enfatizar las conexiones entre ellas (Artigue, 1991; Duval, 2006; Ferrini-Mundy \& Graham, 1994; Tall et al., 2008). En particular, para la comprensión profunda de la integral definida, es necesario que los estudiantes presten atención al proceso de transformar y conectar las distintas representaciones (Camacho et al., 2013, p. 50).

Teniendo en cuenta estos aspectos, la tecnología puede ayudar a crear entornos de aprendizaje dinámicos e interactivos, en los que se utilizan simultáneamente distintas representaciones de los conceptos y se puede observar cómo varían unas con relación a otras. El uso de tecnología como instrumento de mediación semiótica, permite mostrar simultáneamente varias representaciones y favorece la integración entre las representaciones y el dinamismo (Blume \& Heid, 2008; Heid \& Blume, 2008; Lagrange \& Artigue, 2009; Maschietto, 2008; Tall et al., 2008).

Junto a la tecnología, las investigaciones también señalan el papel complementario, pero esencial, que juegan las tareas con lápiz y papel por su valor epistémico (Kieran \& 
Drijvers, 2006), así como el trabajo en pequeño grupo que ayuda a la interacción entre iguales, favoreciendo la dimensión social, y compartiendo ideas en una discusión en clase guiada por el profesor (Ferrara et al., 2006).

El concepto de integral definida necesita de una comprensión del concepto de límite, y algunas investigaciones destacan las dificultades de los estudiantes para comprender la integral debido a que no tienen una comprensión adecuada de este concepto (Boigues et al., 2010; Orton, 1983).

En este trabajo se utiliza la integral de Darboux, que es la que se usa habitualmente en este nivel de enseñanza, aunque muchas veces se la denomina integral de Riemann. En los libros de texto se confunde generalmente las sumas de Darboux y las de Riemann (Porres, 2012; Porres et al., 2017). En la primera, se define la integral superior como límite de sumas superiores y la integral inferior como límite de las sumas inferiores. En estas sumas, se toma respectivamente el ínfimo y el supremo de la función en cada subintervalo como altura de los rectángulos, y como en este nivel se trabaja con funciones continuas, el mínimo y el máximo. En la segunda, se trata de un punto cualquiera del intervalo.

Porres (2012) en su investigación sobre la integral definida en estudiantes de segundo de bachillerato de ciencias sociales afirma que:

La integral de Darboux (al establecer las sumas inferiores y superiores y, de ahí, las integrales inferior y superior de Darboux), es más fácil, completa y precisa que la integral de Riemann (debido a la imprecisión de los puntos intermedios asociados a una partición y a la dificultad en establecer su propia definición) para que los estudiantes de Bachillerato adquieran mejor el concepto de integral definida y, al ser ambas equivalentes. (p. 648).

El objetivo de este artículo es presentar tres tareas propuestas a estudiantes de 1718 años, que se apoyan en applets de Geometría dinámica y en una hoja de cálculo, para trabajar el concepto de integral definida, que se enmarcan en una unidad didáctica sobre la integral definida, e identificar distintos perfiles de estudiantes tras la realización de estas.

\section{Marco teórico}

En esta sección abordamos la concepción dinámica y métrica del límite y los distintos sistemas de representación de los conceptos del Cálculo.

\section{Concepción dinámica y métrica del límite}

Cornu (1991) afirma que los estudiantes suelen aprender los aspectos cognitivos del límite a partir de la idea de aproximación (concepción dinámica), no de su definición 
matemática (concepción métrica). Blázquez y Ortega (2002) definen la concepción dinámica del límite de una función en un punto como una aproximación óptima:

Sea $f$ una función y $a$ un número real, el número $L$ es el límite de la función $f$ en el punto $a[\ldots]$ si cuando $x$ se acerca al número $a$ más que cualquier aproximación, sus imágenes $f(x)$ se acercan a $L$ más que cualquier otra aproximación fijada”. (p. 79).

Esta concepción es relativamente complicada para los estudiantes que deben visualizarla para adquirir la idea formal de límite y una compresión insuficiente de esta concepción dificulta construir la definición formal o concepción métrica: “[...] cuando ' $x$ $a$ ' en valor absoluto se aproxima a $0, ' f(x)-L$ ' en valor absoluto se aproxima a 0 ” (Cottril et al.,1996).

Así pues, la comprensión de la concepción métrica en términos de desigualdades empieza con la construcción de la concepción dinámica, y esta se apoya en el modo de representación numérico (Pons, 2014, Pons et al., 2011; Valls et al., 2011).

Por otra parte, Sealey $(2006,2014)$ afirma que es imprescindible que los estudiantes comprendan las sumas de Riemann y las integrales definidas, entre otras razones porque construir una integral definida apropiada requiere saber qué integrar y la comprensión de estas sumas proporcionará a los estudiantes las herramientas que necesitan.

\section{Integral de Darboux}

Darboux introdujo por primera vez las integrales superior $\mathrm{e}$ inferior correspondientes a las sumas superiores e inferiores de Riemann (Apostol, 1960). Porres (2012) en su epistemología del cálculo integral, utiliza la siguiente definición de integral de Darboux:

Dada una función $f(x)$ acotada en un intervalo $[a, b]$ y una partición $x_{0}, x_{1}, x_{2}, x_{3}, \ldots$, $x_{n-1}, x_{n}$ de dicho intervalo. Sean $m_{i}$ el ínfimo de la función $f(x)$ en el subintervalo $\left[x_{i-1}, x_{i}\right]$ y $M_{i}$ el supremo de la función $f(x)$ en $\left[x_{i-1}, x_{i}\right]$.

Se definen suma inferior y suma superior de la función $f(x)$, en el intervalo [a,b], asociadas a la partición anterior a las expresiones, respectivamente:

$$
\begin{aligned}
& s=m_{1}\left(x_{1}-x_{0}\right)+m_{2}\left(x_{2}-x_{1}\right)+m_{3}\left(x_{3}-x_{2}\right)+\ldots+m_{n}\left(x_{n}-x_{n-1}\right) \\
& S=M_{1}\left(x_{1}-x_{0}\right)+M_{2}\left(x_{2}-x_{1}\right)+M_{3}\left(x_{3}-x_{2}\right)+\ldots+M_{n}\left(x_{n}-x_{n-1}\right)
\end{aligned}
$$

Darboux denomina integral inferior de la función $f(x)$ en el intervalo $[a, b]$ al extremo superior de las sumas inferiores e integral superior al extremo inferior de las 
sumas superiores cuando varían las particiones (denotadas respectivamente por $\int_{a}^{b} f(x) d x$ e $\left.\overline{\int_{a}^{b}} f(x) d x\right)$.

Si las dos integrales coinciden, se dirá que la función $f(x)$ es integrable en el intervalo $[a, b]$ y tal evento se indicará mediante la expresión $\int_{a}^{b} f(x) d x$. (Porres, 2012, Anexo B, Cap. V, p. 81).

\section{Distintos sistemas de representación}

La importancia de usar múltiples representaciones para la construcción de conceptos de Cálculo se ha puesto de manifiesto en numerosas investigaciones, como en el trabajo de Sánchez-Matamoros et al., (2006) sobre el desarrollo del esquema de derivada. También para favorecer la comprensión del concepto de integral, así como enfatizar las conexiones entre ellas para llegar a una comprensión más profunda del mismo (Artigue, 1991; Duval, 2006; Ferrini-Mundy \& Graham, 1994; Tall et al., 2008).

Pero algunos trabajos muestran que los estudiantes no relacionan las aproximaciones gráficas y analíticas de los conceptos (Ferrini-Mundy \& Graham, 1994) y muestran reticencias para utilizar métodos gráficos (Dreyfus \& Eisenberg, 1986; Dreyfus, 1991). Además, cuando se quiere calcular el área de la superficie bajo una curva mediante las sumas de Riemann, los estudiantes en algunas ocasiones no son capaces de interpretar el proceso de aproximación al área mediante las aproximaciones numéricas (Camacho et al., 2013).

El uso de la tecnología permite a los estudiantes liberarse de cálculos tediosos. Ferrara et al., (2006) sugieren usar la tecnología para tratar la integral en primer lugar a nivel numérico y gráfico como sumas de rectángulos de base cada vez menor, y después a nivel simbólico. Por otra parte, representar gráficamente los conceptos del Cálculo y dar dinamismo a las imágenes puede ser "parte constitutiva del pensamiento más que una mera ayuda o simplificador para llevar a cabo la tarea matemática" (Swidan \& Yerushalmy, 2014, p. 530). Cabe también señalar el potencial del uso de hojas de cálculo y programas de cálculo simbólico (Hong \& Thomas, 1997).

Duval $(1993,2006)$ señala que, para la formación del concepto, es absolutamente necesario la interacción entre las distintas representaciones y que para construir el 
significado de los objetos matemáticos es necesaria la coordinación interna entre representaciones.

\section{Diseño de tareas que se apoyan en applets}

Uno de los problemas fundamentales de las matemáticas ha sido el cálculo del área de una superficie (Guzmán, 2010), y ha estado en el origen del Análisis Matemático. Courant y Robbins (2002, p. 440) afirman que "El primer concepto básico del Análisis Matemático es el de integral".

En el currículum español de $2^{\circ}$ de Bachillerato (17-18 años) se inicia el estudio del Cálculo Integral con el cálculo de primitivas y posteriormente se trata la integral definida. Pero la unidad didáctica en que vamos a apoyar nuestro estudio ${ }^{1}$ se inicia con la integral definida, previamente al cálculo de primitivas. Y se hace a partir del cálculo del área bajo una curva (Turégano, 1998), primando así su génesis histórica. Parte del cálculo del área de un círculo por el método de exhaución, después plantea situaciones en las cuales el área bajo la curva representa distintas magnitudes como las precipitaciones totales en un periodo de tiempo, la distancia recorrida a partir de la gráfica de la velocidad etc. tareas para aproximar el área y finalmente define la integral como el límite de las sumas de Darboux. A continuación, se tratan las propiedades de la integral, se introduce la función integral, el Teorema fundamental del cálculo y, por último, la Regla de Barrow y ejercicios de aplicación (Figura 1).

Figura 1: Índice de la unidad didáctica integral definida en el libro "Matemáticas $2^{\circ}$ Bachillerato"

\begin{tabular}{|l|l|}
\hline INTEGRAL DEFINIDA & $\begin{array}{l}\text { Integración de funciones racionales } \\
\text { Ampliación: integración d efunciones irracionales } \\
\text { Estimación del área }\end{array}$ \\
$\begin{array}{l}\text { Integral definida } \\
\text { Ampliación: funciones trigonométricas }\end{array}$ \\
$\begin{array}{l}\text { Teorema fundamental del cálculo integral } \\
\text { Regla de Barrow }\end{array}$ & $\begin{array}{l}\text { APLICACIONES DE LA INTEGRAL } \\
\text { Cambio de variable en la integral definida }\end{array}$ \\
PRIMITIVAS. CÁLCULO DE PRIMITIVAS & Cálculo de áreas: áreas sobre ele eje de abscisas \\
Introdución & Cálculo de áreas: áreas sobre el eje de ordenadas \\
Integrales inmediatas región comprendida entre dos curvas \\
Cambio de variable & fy $g$ \\
Integración por partes & Volumen de un cuerpo de revolución \\
\hline
\end{tabular}

Fuente: Las autoras (2020)

1 Botella, L. M., Cascón, B., Martín, C., Millán, L. M., Moltó, C., Pérez, C. y Salinas, E (2001). Matemàtiques 2. Alcoi: Marfil. 
El método de trabajo de este libro de texto está basado en la resolución de problemas. Cada unidad didáctica se inicia con una actividad de introducción al tema, después problemas para introducir los conceptos, posteriormente estos se formalizan y al final se plantean actividades de aplicación y consolidación (Azcárate et al., 1997).

De la unidad didáctica hemos seleccionado una tarea que hemos desglosado en tres que describimos a continuación. De acuerdo con propuestas de las investigaciones, como la aproximación al proceso histórico del desarrollo del concepto de integral (Azcárate et al., 1996; Ferrara et al., 2006), las hemos transformado en tareas a realizar con el apoyo del applets de geometría dinámica y una hoja de cálculo, para aproximar las áreas por métodos geométricos y series numéricas, e introducir el límite de manera formal al final (Aranda y Callejo, 2010b).

Las tareas se realizaron por parejas, dado que el trabajo en pequeños grupos y la discusión y uso simultáneo de representaciones geométricas dinámicas e interactivas puede facilitar la construcción del concepto (Ferrara et al., 2006). Usaremos tres tipos de representaciones: geométricas, analíticas y numéricas.

\section{Tarea del libro de texto}

A continuación se muestra el problema número 50 del libro, Área bajo una parábola (Figura 2).

Figura 2: Problema 50 del libro de texto

L'objectiu és tractar de calcular amb la major exactitud possible l'àrea de la regió del plànol compresa entre la gràfica de la corba $y=x^{2}$ i les rectes $x=0$ i $x=5$.

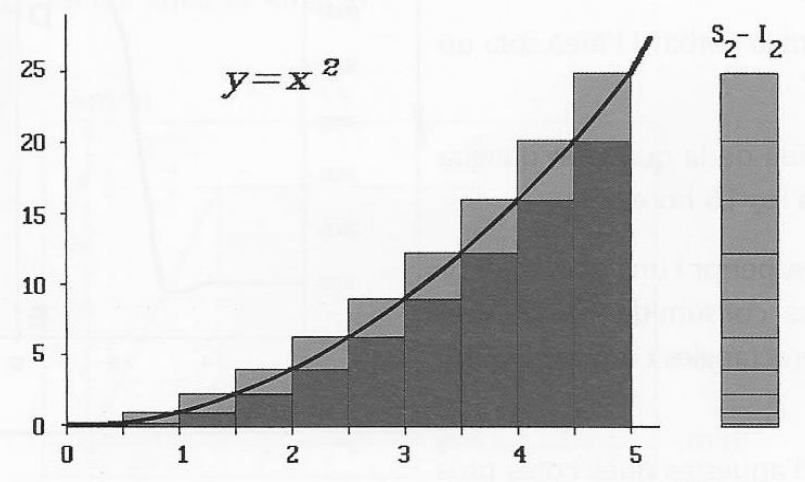

Amb la utilització del mètode de rectangles, i prenent-los en principi de base 1 , entre quins valors es troba l'àrea perduda? I si els prenem de base 0'5? Quan obtindríem el valor exacte d'aquesta àrea?

Fuente: Las autoras (2020) 
Como se puede ver en el problema propuesto en el libro de texto, se pretende calcular el área de la región limitada por la gráfica de la función $y=x^{2}$, el eje de abscisas y las rectas $x=0$ y $x=5$. Para ello se utilizan, sin nombrarlas, las sumas inferiores $\mathrm{y}$ superiores de Darboux, correspondientes a rectángulos cuyas alturas son respectivamente el mínimo y el máximo de la función en cada subintervalo. Se inicia con 5 subintervalos, después con 10, y pregunta cuando se obtendría el valor exacto del área. A la derecha de la figura se muestra un rectángulo formado por la acumulación de las diferencias, cuya área es la diferencia entre las sumas superiores y las inferiores.

De esta forma, se está tratando con la integral como límite de las sumas de Darboux, mediante la concepción dinámica del límite en las aproximaciones al área de las sucesiones de las sumas inferiores y superiores, y mediante la concepción métrica al trabajar con el área del rectángulo de acumulación de las diferencias entre las sumas, al preguntar cuando se obtendría el valor exacto.

A partir de esta tarea del libro de texto se diseñaron tres para tratar: el concepto de integral como límite; el cálculo del área bajo un arco de parábola y el error de la aproximación. Presentamos las tres tareas, y las hipótesis sobre el proceso de aprendizaje de la primera tarea, por una parte, y de las dos siguientes juntas por otra.

\section{Concepto de la integral como límite: Área del cuadrante}

Esta tarea se apoya en un applet $^{2}$ de la Figura 3.

Figura 3: Pantalla del applet de la tarea Área del cuadrante

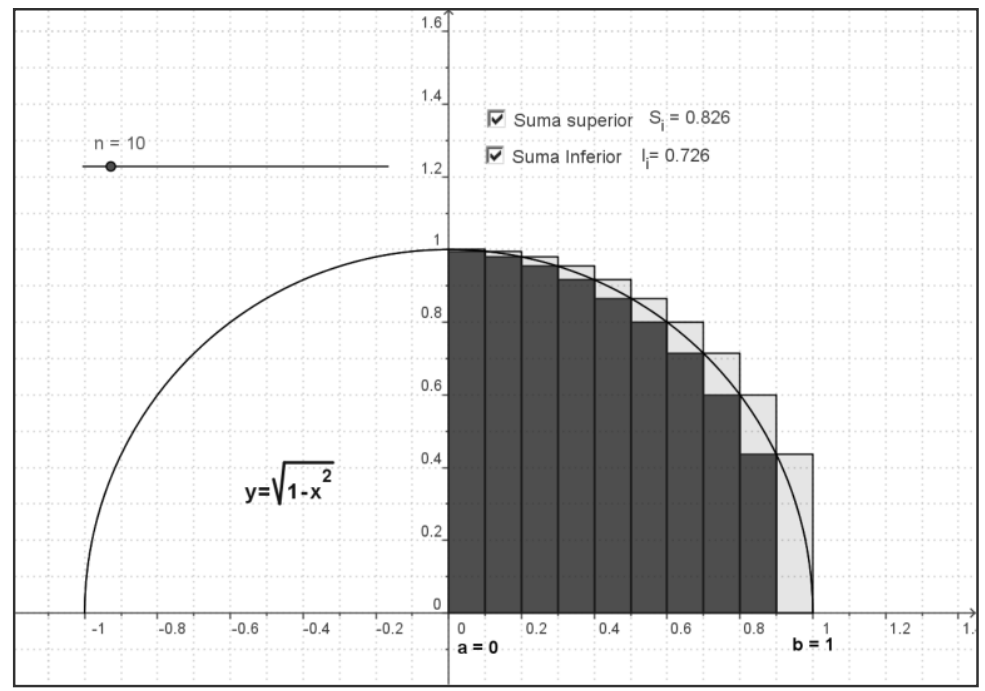

Fuente: Las autoras (2020)

\footnotetext{
${ }^{2}$ El applet se puede consultar en: https://www.geogebra.org/m/zYgruzB6.
} 
En este applet está dibujada media circunferencia de radio 1, y en el primer cuadrante se muestran las sumas superiores e inferiores en lenguaje gráfico (rectángulos superiores e inferiores de igual base) y numérico $\left(S_{i}, I_{i}\right)$. Los estudiantes pueden variar el número de rectángulos de las sumas superiores e inferiores desde 1 hasta 100 mediante un deslizador ( $n$ ) situado en la parte superior izquierda.

En esta tarea los estudiantes conocen el valor del área, dado que es un cuadrante de círculo. Para construir el concepto de integral como límite, se les pide que aproximen el área bajo la curva $y=\sqrt{1-x^{2}}$ en el intervalo [0,1] utilizando el applet diseñado ad hoc (Figura 3), en el que pueden modificar el número de subintervalos (n) de la partición desde 1 hasta 100, todos ellos de la misma longitud, y se muestran gráfica y numéricamente las sumas de Darboux. Con ello se pretende que los estudiantes se den cuenta de que al aumentar el valor de $n$ se obtiene una mejor aproximación al área. Después se les pide el valor de n para aproximar el área con un error menor que un número dado, y por último, que relacionen $\mathrm{n}$ con las aproximaciones y con el error de éstas. Tratamos así las aproximaciones dinámica y métrica del límite, y relacionamos ambas, con el objetivo de que los estudiantes coordinen estas aproximaciones.

Posteriormente, se les pide que completen las celdas vacías correspondientes a $n=3$, $n=4$ y $n=5$ en una hoja de cálculo ya diseñada (Figura 4) y en la que aparecen los valores correspondientes a $n=1$ y $n=2$. Una vez completados, se les pide que comparen estos valores con los obtenidos en el applet. Con ellos se pretende que reflexionen sobre lo que ocurre al variar $n$, y que la tarea no se convierta en un mero ejercicio de manipulación.

Figura 4: Vista de la hoja de cálculo de la tarea Área del cuadrante

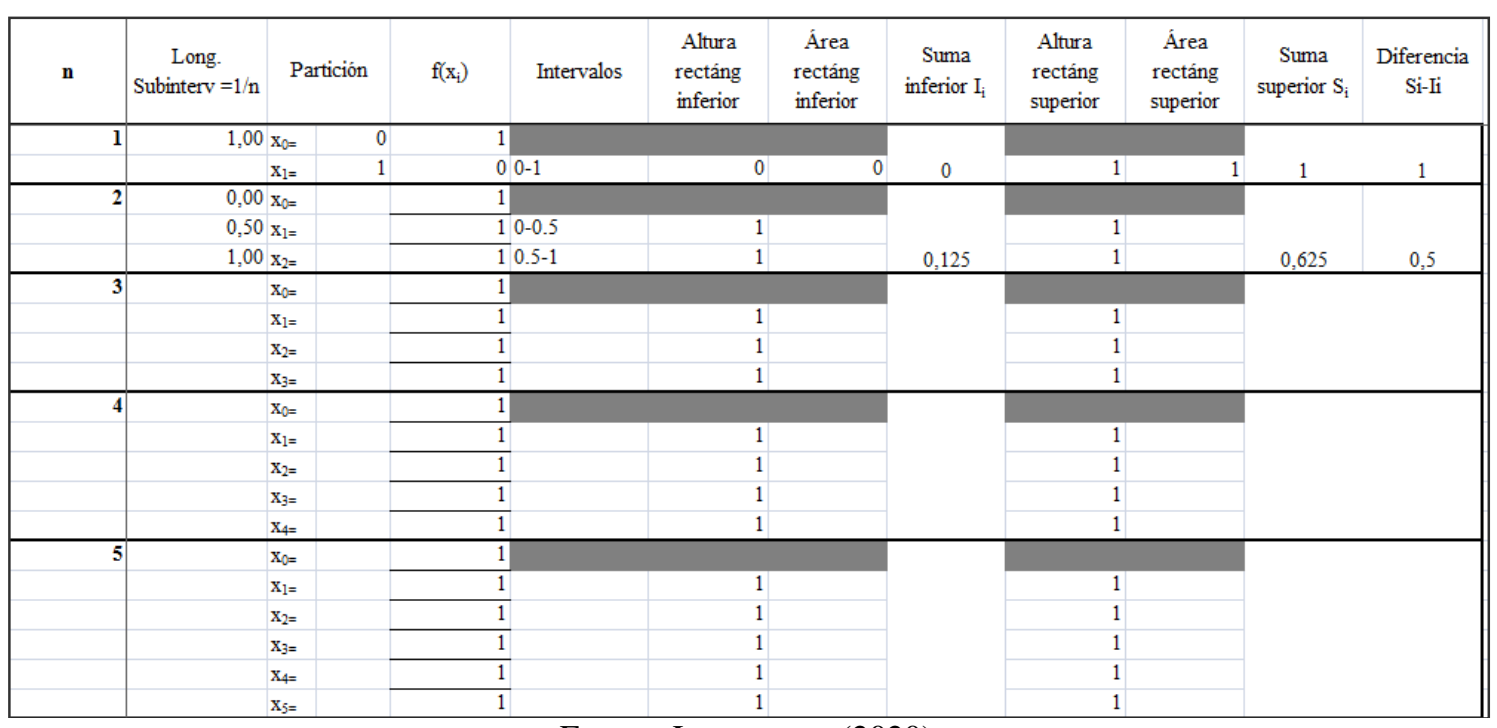

Fuente: Las autoras (2020) 
La hipótesis sobre el proceso de aprendizaje es que cuando los estudiantes aumenten el valor de $\mathrm{n}$ y aumenten el número de subintervalos de la partición les permitirá relacionar el incremento del número de subintervalos con el valor de las sumas superiores e inferiores, el recubrimiento del cuadrante del círculo mediante rectángulos y una cota del error de la aproximación. También podrán inferir que al aumentar el valor de $\mathrm{n}$ obtienen una aproximación mejor y que el error se mantiene menor que un valor determinado dado previamente. Estos procesos y el uso simultáneo de distintos sistemas de representación les pueden ayudar a realizar las coordinaciones necesarias para la construcción de las aproximaciones dinámica y métrica del límite y la coordinación de ambas.

\section{Significado de las sumas de Darboux: Cálculo del área bajo un arco de parábola}

Esta tarea se apoya en un applet $^{3}$ de la Figura 5 en el que pueden modificar el número de subintervalos, pueden exponer/ocultar el valor de la longitud de los subintervalos, las sumas inferiores y superiores y el mínimo o máximo de cada subintervalo y la diferencia entre ambas.

En el applet (Figura 5) se muestra un arco de la parábola $y=x^{2}$, hay un deslizador para variar el número de subintervalos $(n)$ desde 1 hasta 100 , y casillas para que se muestren/oculten los puntos de la partición del intervalo [0,1], el mínimo de cada subintervalo, la suma inferior, el máximo de cada subintervalo, la suma superior y el área, en lenguaje gráfico y numérico.

Figura 5: Pantalla del applet 'Cálculo del área bajo un arco de parábola'

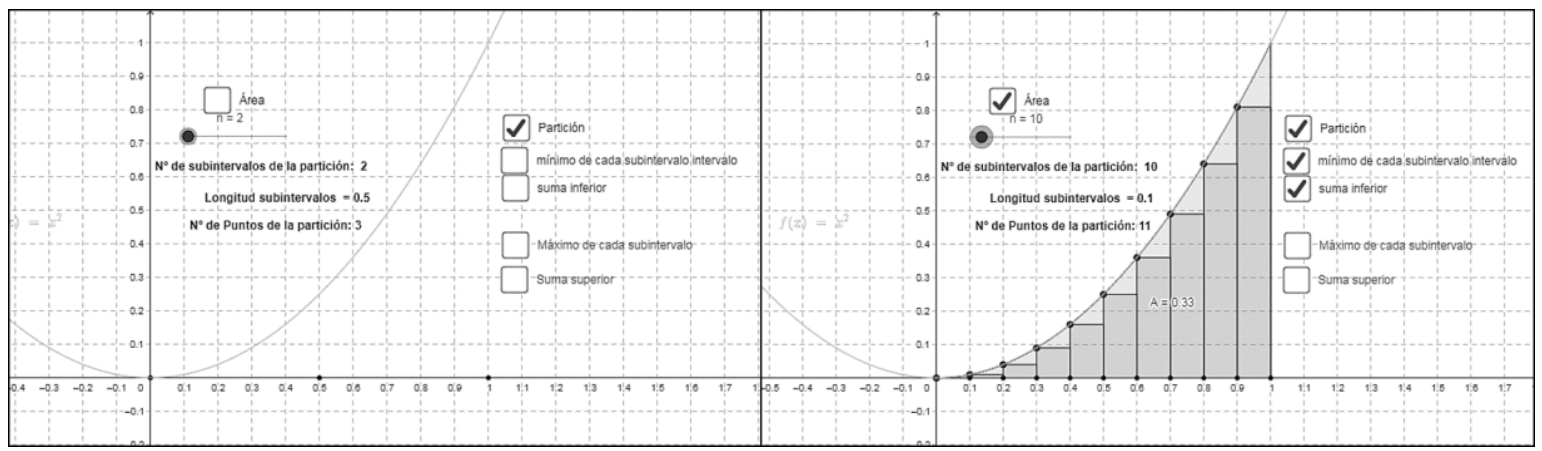

Fuente: Las autoras (2020)

En esta tarea los estudiantes, buscan una aproximación al área bajo un arco de la parábola $y=x^{2}$, en el intervalo [0,1]. A diferencia de la tarea del cuadrante de círculo, los estudiantes no conocen el valor de esta área.

\footnotetext{
${ }^{3} \mathrm{El}$ applet se puede consultar en: https://www.geogebra.org/classic/aratwva4.
} 
El objetivo de esta tarea es que los estudiantes den significado a la expresión en lenguaje analítico de las sumas de Darboux para aproximar el área coordinando las representaciones analíticas y geométricas. Para ello, se les pregunta qué significa $n$ (número de subintervalos), la relación entre $n$, el número de puntos de la partición y la longitud de los subintervalos. Después se les pide que escriban los valores de los puntos extremos de la partición para $n=1,2,3,4$ y 10 y para cualquier valor de $n$. También se les pide que indiquen cual es la altura de los rectángulos en el caso de las sumas inferiores y superiores. Y, por último, que busquen fórmulas para las sumas inferiores y superiores.

\section{Parábola. Error de la aproximación}

En esta tarea se trata de calcular el error de la aproximación al área. Se apoya en el applet $^{4}$ de la Figura 6, que se diferencia del de la tarea anterior (Figura 5) en que se ha añadido el rectángulo de acumulación de las diferencias entre las sumas superiores e inferiores.

Figura 6: Pantalla del applet 'Parábola. Error de la aproximación'

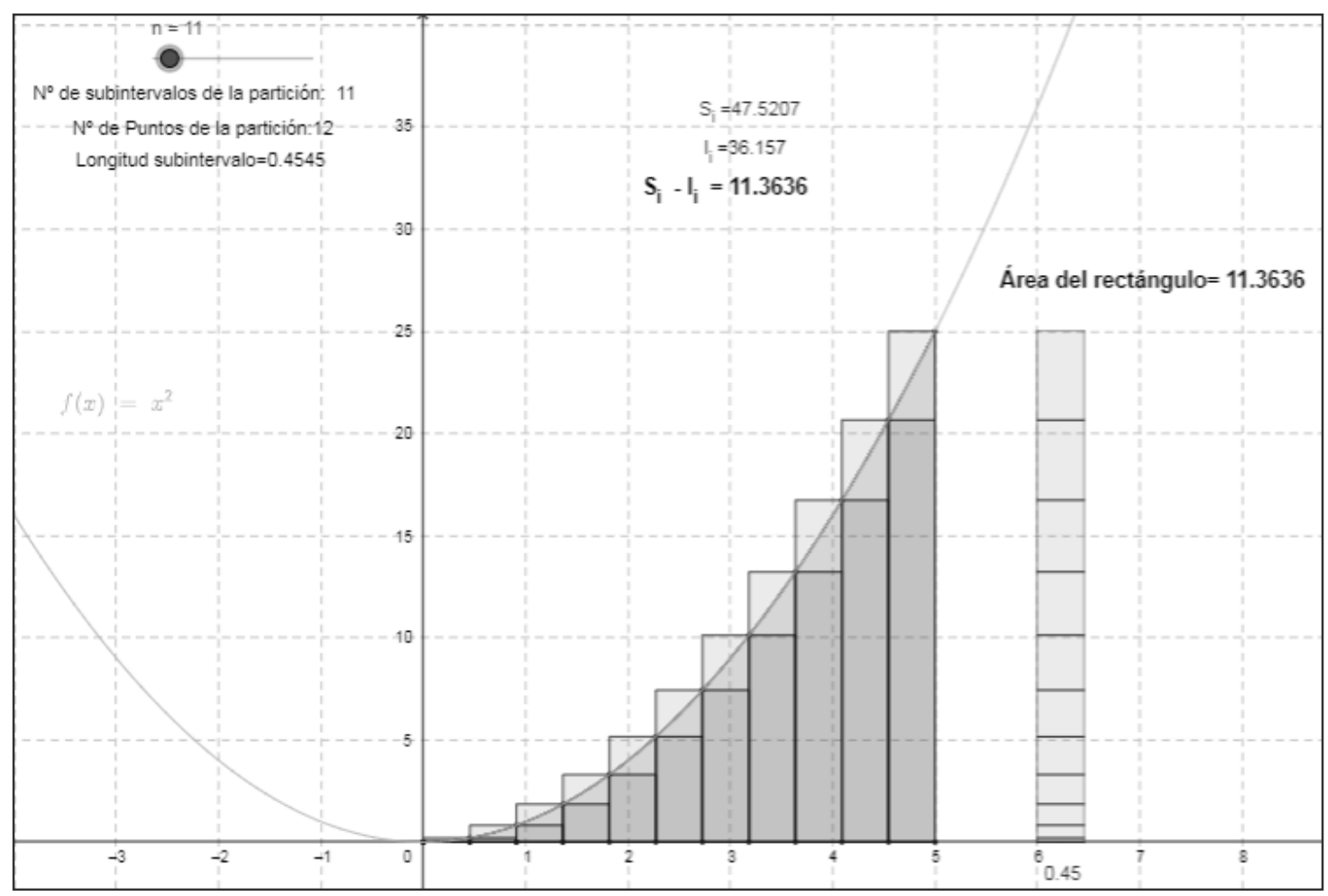

Fuente: Las autoras (2020)

El objetivo de esta tarea es seguir ayudando a los estudiantes a coordinar distintos sistemas de representación: el geométrico, el verbal y el analítico, tanto para las sumas de

\footnotetext{
${ }^{4} \mathrm{El}$ applet se puede consultar en: https://www.geogebra.org/m/TKWE3v8u.
} 
Darboux como para la diferencia entre ellas. Así podrán identificar el número de subintervalos para que el error de la aproximación sea menor que un valor dado.

Para ello, se les pide que experimenten variando el número de subintervalos $(n)$ y observen lo que ocurre con los rectángulos de acumulación de diferencias entre las sumas superiores e inferiores y con el valor numérico de esta diferencia, que busquen una fórmula para el área del rectángulo de las diferencias, y para ello se fijen en si la base o la altura de este rectángulo depende de $n$, calculando estas para $n=10,20,50$ y $n$. Posteriormente que obtengan la fórmula para las diferencias entre sumas superiores e inferiores, que relacionen el área del rectángulo de diferencias con el error máximo cometido al aproximar el área. Y por último, que busquen el número de subintervalos para que el error sea menor que dos valores dados $(0,1$ y 0,01), y para un error cualquiera. En la Figura 7 se muestra estas cuestiones propuestas a los estudiantes.

Figura 7: Tarea Parábola: Error de la aproximación

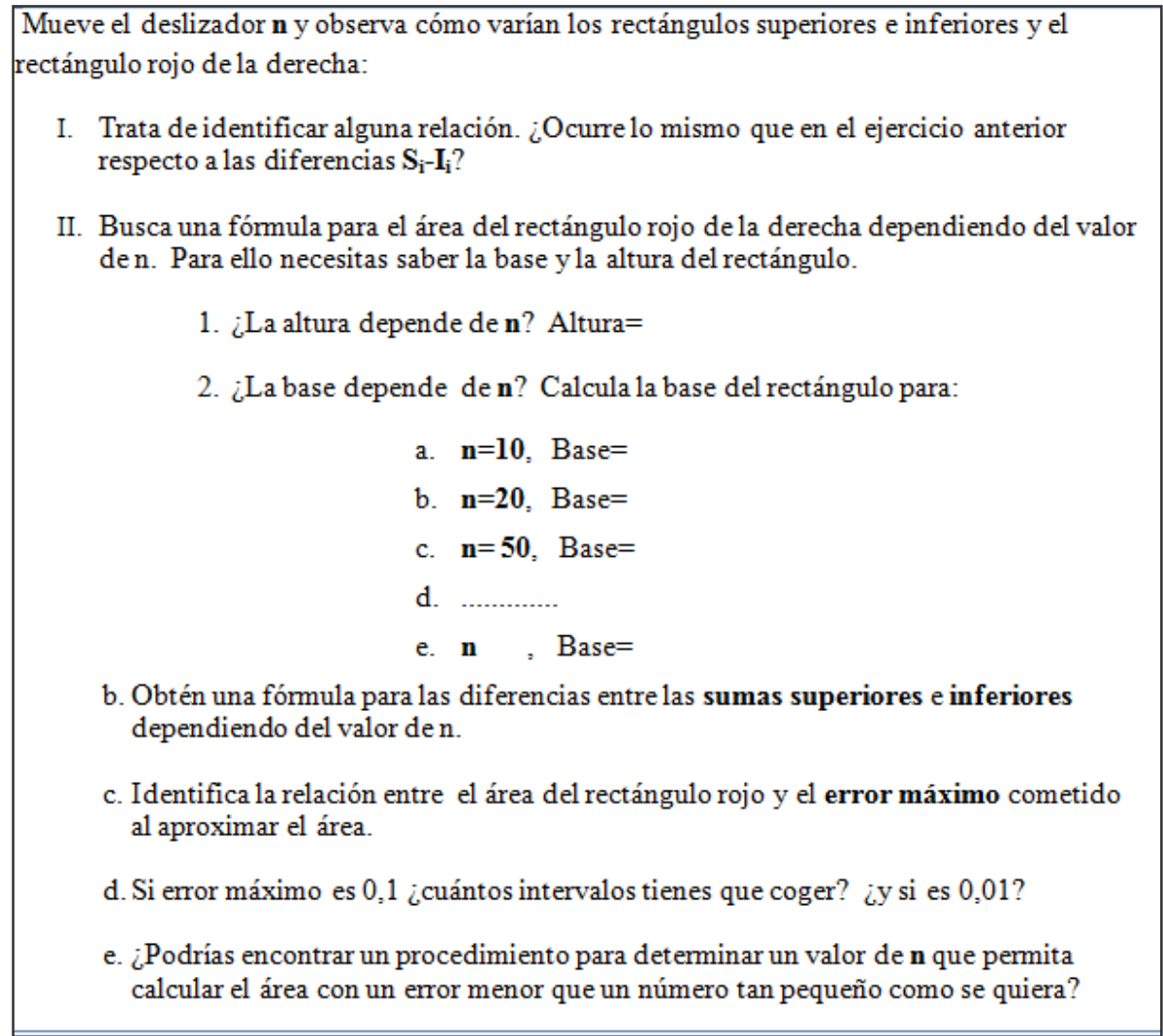

Fuente: Las autoras (2020)

La hipótesis sobre el proceso de aprendizaje de las dos últimas tareas es que los estudiantes, al experimentar aumentando el valor de $n$, número de subintervalos de la partición, y observar lo que ocurre con la base y altura de los rectángulos, y las imágenes de los valores extremos de la partición, podrán relacionar el valor numérico de las sumas 
superiores e inferiores con la suma de las áreas de los rectángulos por defecto y por exceso y podrán inferir que al aumentar el valor de $n$ el error de la aproximación es menor. Esta inferencia les podrá ayudar a coordinar distintos sistemas de representación.

Por una parte el geométrico, el verbal y el algebraico en la tarea Parábola en las sumas superiores e inferiores: rectángulos que aproximan el área y fórmula para las sumas en función de $n$. En la tarea Parábola, error de la aproximación, los rectángulos de diferencias entre los rectángulos por exceso y por defecto, y la fórmula de estas diferencias. Así podrán obtener el valor de $n$ para que el error sea menor que un valor dado y extender estas coordinaciones para cualquier error.

\section{Aplicación de las tareas}

\section{Participantes}

Los participantes fueron 15 estudiantes de $2^{\circ}$ de Bachillerato. Trabajaron durante ocho sesiones de una hora en un aula de informática, por parejas y un trío, con las guías de trabajo y con los applets. Disponían también de calculadoras. En cada sesión se proponían las tareas, que iban resolviendo a su ritmo. Algunas parejas no completaron todas las tareas, pero al inicio de la sesión siguiente se hacía una puesta en común y se institucionalizaba el nuevo conocimiento.

Durante las sesiones, escribían sus respuestas en las hojas de tareas y también se registraba en archivos de vídeo y audio mediante el programa CamStudio (Codes et al., 2007) todo lo que hacían en el ordenador y sus diálogos.

\section{Recogida y análisis de datos}

Los datos de la investigación son las respuestas escritas en las hojas de tareas, los registros de las acciones que efectuaron con los applets para resolver las tareas y los diálogos entre ellos mientras las resolvían, que quedaron registrados con el programa CamStudio.

El análisis de datos se realizó en tres fases. En la primera, se hizo la transcripción de las comunicaciones orales de las sesiones que se consideraron de interés para la investigación y se fueron ilustrando con las capturas de pantalla indicando las acciones realizadas con los applets. En la segunda se identificaron los avances de los estudiantes en relación con los objetivos de cada tarea, y del proceso de aprendizaje. En tercer lugar, se 
describieron perfiles de los estudiantes en relación con la coordinación de las aproximaciones dinámica y métrica del límite y entre distintos sistemas de representación.

En la segunda fase del análisis se identificaron aquellas comunicaciones orales y registros de interacciones en que se evidenciaba los saltos cognitivos que se producen tras realizar las tareas. Estas acciones son: relacionar, inferir, coordinar y extender.

Por ejemplo, la pareja K-MA en la construcción de la integral como límite, experimentó con el applet y relacionó el incremento del número de subintervalos de la partición con el valor de las sumas inferiores y superiores y el recubrimiento de la superficie del cuadrante mediante rectángulos. Esta relación les permitió inferir que al aumentar el número de rectángulos se obtenía una mejor aproximación del área y así pudieron coordinar los procesos de aproximación de las sucesiones de las sumas en el dominio (número de subintervalos) y en el rango (valores de las sumas), es decir la concepción dinámica del límite y también coordinar la representación geométrica (superficie del cuadrante) con la representación numérica (valores de la sucesión de las sumas inferiores y superiores). En la Figura 8 mostramos el protocolo de una pareja, KMA, resolviendo una cuestión de la tarea área del cuadrante. Los estudiantes coordinaron el error de las aproximaciones mediante las sumas inferiores y las sumas superiores, así como la monotonía de estas sucesiones.

Figura 9: Protocolo de la pareja K-MA resolviendo la cuestión III de la tarea 'Área del cuadrante'

\begin{tabular}{|c|c|c|}
\hline Interacciones con el applet & Comunicaciones orales & Acciones \\
\hline $\begin{array}{l}\text { Dejan marcada la casilla de las } \\
\text { sumas superiores. } \\
\text { Aumentan } n \text { de } 1 \text { a } 100 \text {. }\end{array}$ & $\begin{array}{l}\text { [29] MA: Y con la siguiente [se refieren a esta tarea] } \\
\text { pasa lo mismo, sólo que ésta es con la... } \\
\text { [30] K: Sólo que va disminuyendo... va } \\
\text { disminuyendo ésta. }\end{array}$ & \\
\hline $\begin{array}{l}\text { Parten de } n=1 \\
\text { Marcan también sumas inferiores } \\
\text { Mueven el deslizador } n=4,6, \ldots 100 \text {. } \\
\text { Aplican el zoom para ampliar. }\end{array}$ & 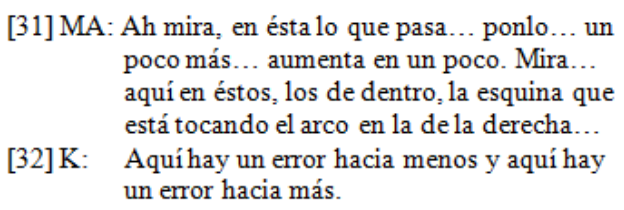 & $\begin{array}{l}\text { Coordinan el error de } \\
\text { ambas aproximaciones }\end{array}$ \\
\hline$n=1, n=13$ & $\begin{array}{l}\text { [33] MA: Esta se va acercando desde arriba, sabes, o } \\
\text { sea, empieza desde un valor y va } \\
\text { disminuyendo... } \\
\text { [34] K: Eso digo, que aquí va aumentando [se refiere } \\
\text { a las sumas inferiores] y aquí disminuyendo } \\
\text { [serefiere a las sumas superiores]. }\end{array}$ & $\begin{array}{l}\text { Coordinan la } \\
\text { monotonía de las } \\
\text { sucesiones delas } \\
\text { sumas superiores e } \\
\text { inferiores }\end{array}$ \\
\hline $\begin{array}{l}n=100 \\
\text { Disminuyen el valor de } n \text { y luego } \\
\text { vuelven al valor } n=100 \text {. }\end{array}$ & $\begin{array}{l}\text { [35] MA: Aquí aumenta hasta } \pi / 4 \text {, claro. } \\
\text { [36] MA: Pongo que aquí se aproxima aumentando. }\end{array}$ & \\
\hline
\end{tabular}

Fuente: Aranda (2015, pp. 95-96) 


\section{Resultados}

Esta sección presentamos en primer lugar los perfiles encontrados en relación con la construcción de la aproximación al área como límite de una sucesión y en segundo lugar en relación con el significado de las sumas de Darboux y el error de la aproximación.

\section{Perfiles con relación a la construcción de la integral como límite}

Se identificaron tres perfiles en relación con la construcción de la aproximación al área bajo una curva (Aranda, 2015; Aranda \& Callejo, 2017a) a partir de la tarea Área del cuadrante.

Los estudiantes del perfil 1 experimentaron con el applet para intentar realizar la tarea, y fueron capaces de relacionar el número de intervalos de la partición y las sumas de las áreas de los rectángulos por defecto y por exceso, como se muestra en la Figura 9, respondiendo al apartado III de la tarea "área del cuadrante":

Figura 9: Protocolo de la pareja A-J resolviendo la cuestión III de la tarea 'Área del cuadrante'

\begin{tabular}{|c|c|}
\hline \multicolumn{2}{|c|}{$\begin{array}{l}\text { III. Deja sólo marcada la casilla de las sumas superiores. Da a } \mathrm{n} \text { el valor } 1 \text { y ve } \\
\text { aumentando } n \text {. Observa lo que ocurre con el valor de esta suma. Escribe lo que has } \\
\text { observado. }\end{array}$} \\
\hline Interacciones con el applet & Comunicaciones orales \\
\hline $\begin{array}{l}\text { Dejan marcada sólo la casilla de la suma } \\
\text { superior. } \\
\text { Aumentan } n \text { poco a poco hasta llegar a } \\
n=100, n=97, n=100\end{array}$ & $\begin{array}{l}\text { [22] J: Podemos comprobar que la suma } \\
\text { superior va disminuyendo cada vez } \\
\text { más lentamente, hasta llegar a suma } \\
\text { superior } n=100 \text {, que queda suma } \\
\text { superior, } \mathrm{S}_{\mathrm{i}}=0.790 \text {. Empieza de } 1 \mathrm{y} \\
\text { baja a } 0.790 \text {. La progresión cada } \\
\text { vez que } n \text { aumenta, la disminución } \\
\text { es más lenta debido al número de } \\
\text { intervalos que hay. }\end{array}$ \\
\hline
\end{tabular}

Fuente: Aranda (2015, p. 111)

Pero no mostraron evidencias de relacionar el incremento del número de subintervalos con que las sucesiones de las sumas superiores e inferiores convergen en un mismo valor, es decir con la cota del error de la aproximación, pues cuando se les pidió el valor del área con un error menor que 0.02, como habían obtenido que para 100 subintervalos el error es menor que 0.1 , relacionaron estos dos valores, 0.02 y 0.1 , notaron 
que a un menor error debía corresponder un número mayor de subintervalos y pensaron que la relación era de proporcionalidad inversa, como se muestra en la Figura 10. Pero no mostraron evidencias de la comprensión de la concepción métrica del límite.

Figura 10: Protocolo de la pareja A-J resolviendo la cuestión IV. b de la tarea 'Área del cuadrante'

\begin{tabular}{|c|c|}
\hline \multicolumn{2}{|c|}{ IV. b. Y si el error máximo fuera 0.02 , ¿cuál sería el valor del área y de $n$ ? } \\
\hline Interacciones con el applet & Comunicaciones orales \\
\hline $\begin{array}{l}n=1 \text {; van aumentando hasta } n=45 \mathrm{y} \\
\text { después hasta } 100 \text {. } \\
\text { De nuevo a } n=1 \text { y a } 100 . \\
\text { Quitan marcas de la suma inferior y la } \\
\text { superior y las vuelven a poner. } \\
n=43, n=98 \\
\text { Amplian mucho la imagen, sólo se ve un } \\
\text { trozo. Vuelven al zoom normal. }\end{array}$ & $\begin{array}{l}\text { [108] A: Si el error máximo fuera } 0,02, \\
\text { para que sea cinco veces más } \\
\text { grande que } 100, \text { o sea } 500 \text {. ¿Cuál } \\
\text { sería el valor del área y de } n \text { ? Lo } \\
\text { que no sé es si está bien. Pero } \\
\text { mira, si tenemos } 0,02 \text { y lo } \\
\text { multiplicamos por } 5 \text {, nos da } 0,1 \text {, } \\
\text { que } 0.1 \text { era } 100, \text { lo que pasa es que } \\
\text { aquí era error menor y aquí es } \\
\text { error máximo que es la diferencia } \\
\text { que yo no he acabado de pillar. } \\
\text { Pero el valor del área sería... } n \\
\text { igual a quinientos... el valor del } \\
\text { área sería inferior a quinientos } \\
\text { ¿no?, no, superior a quinientos y } n \\
\text { sería quinientos }\end{array}$ \\
\hline
\end{tabular}

Fuente: Aranda (2015, p. 112)

En el segundo perfil los estudiantes mostraron evidencias de relacionar los valores numéricos de las sucesiones con el límite de estas, coordinando la representación numérica (valores de las sucesiones) con la geométrica (recubrimiento de la superficie del cuadrante mediante rectángulos) como se muestra en la Figura 11. 
Figura 11: Protocolo de la pareja K-MA resolviendo la cuestión II de la tarea 'Área del cuadrante'

\begin{tabular}{|c|c|}
\hline \multicolumn{2}{|c|}{$\begin{array}{l}\text { II. Deja sólo marcada la casilla de las sumas inferiores. Da a } n \text { el valor } 1 \text { y ve } \\
\text { aumentando } n \text {. Observa lo que ocurre con el valor de esta suma. Escribe lo que has } \\
\text { observado. }\end{array}$} \\
\hline Interacciones con el applet & Comunicaciones orales \\
\hline $\begin{array}{l}\text { Van moviendo el deslizador: } n=6,2,1,95 \text {, } \\
56 \\
\text { Dejan marcada la casilla de las sumas } \\
\text { inferiores }\end{array}$ & $\begin{array}{l}\text { [16] MA: La suma de los... de los } \\
\text { rectángulos, cuando más } \\
\text { aumenta } n \text { se acerca más al } \\
\text { área real de este trozo, de... o } \\
\text { sea del sector circular. }\end{array}$ \\
\hline $\begin{array}{l}\text { Obtienen una aproximación con la } \\
\text { calculadora del valor del área del cuadrante } \\
\text { de circulo }(\pi / 4) \text {. }\end{array}$ & $\begin{array}{l}\text { [26] K: Pues eso que la suma va } \\
\text { aumentando a... cuanto mayor } \\
\text { es } n \text { y cada vez acercándose más } \\
\text { al área... al área del cuadrante, } \\
\text { ¿no? }\end{array}$ \\
\hline
\end{tabular}

Fuente: Aranda (2015,p.106)

Estos estudiantes notaron que a mayor número de subintervalos la aproximación es mejor, infiriendo que el error es una cota, como se muestra en la Figura 12, al afirmar: "pues a partir de $n=6$ ¿no?

Figura 12: Protocolo de la pareja K-MA resolviendo la cuestión IV de la tarea 'Área del cuadrante'

\begin{tabular}{|c|c|}
\hline \multicolumn{2}{|c|}{$\begin{array}{l}\text { IV. Si se pide el valor del área con un error menor que } 0,1 \text {, ¿cuál sería el valor del } \\
\text { área? ¿Cuál sería el valor de } n \text { para aproximar el área hasta el valor anterior? }\end{array}$} \\
\hline Interacciones con el applet & Comunicaciones orales \\
\hline $\begin{array}{l}n=100 \\
\text { Señalan en la imagen } \mathrm{S}_{\mathrm{i}}=0,790 \mathrm{I}_{\mathrm{j}}=0,780 \\
\text { Mueven el deslizador a } n=47, n=28, n=30 \text {, } \\
n=100\end{array}$ & $\begin{array}{l}\text { [44] MA: Tiene que acercarse a esto } \\
\text { [se refieren a } 0.78 \text { ] con } \\
\text { menos de una décima de } \\
\text { diferencia. } \\
\text { [45] K: O sea, más de } 0.68 \text { y menos } \\
\text { de } 0.88 \text {, }\end{array}$ \\
\hline $\begin{array}{l}\text { Mueven el deslizador } n=1,7,6 \text {; } \\
\text { Señalan que para } n=6 \text { : } \mathrm{S}_{\mathrm{i}}=0.849 ; \mathrm{I}_{\mathrm{j}}=0.682\end{array}$ & [46] K: Pues a partir de $n=6$ ¿no? \\
\hline
\end{tabular}

Fuente: Aranda (2015, p. 108)

Pero no pusieron de manifiesto la conexión entre la concepción métrica, cota del error de la aproximación, con la dinámica, idea de aproximación al límite, ni manifestaron una vinculación entre las aproximaciones al límite y la disminución del error. 
Los estudiantes del perfil 3 mostraron evidencias de relacionar los valores numéricos de las sucesiones de sumas inferiores/superiores con el límite de la sucesión, concepción dinámica del límite, coordinando los valores de estas sumas con las superficies del cuadrante del círculo, es decir, coordinando las representaciones numérica y geométrica. También muestran evidencias de la construcción de la concepción métrica de límite al afirmar que a partir de un determinado valor de $n$ la diferencia entre el valor de las sumas superiores/inferiores y el límite es menor que un valor dado previamente. Pero no mostraron evidencias de coordinar ambas concepciones.

\section{Perfiles en relación con el significado de las sumas de Darboux y el error de la aproximación}

También se identificaron tres perfiles en relación al significado de la expresión de las sumas de Darboux (Aranda, 2015).

En el perfil 1 los estudiantes no presentaron evidencias de expresar las sumas de Darboux en ningún sistema de representación ni coordinación entre representaciones. No fueron capaces de expresar en lenguaje analítico las alturas de los rectángulos relacionándolas con los valores de los extremos de los subintervalos de la partición ni llegaron a expresar en lenguaje verbal las sumas inferiores ni superiores.

En el perfil 2, los estudiantes muestran evidencias de coordinar las representaciones para las sumas inferiores y superiores, pero no entre las representaciones de la acumulación de diferencias. Fueron capaces de expresar verbal y analíticamente de forma correcta las sumas inferiores y superiores coordinando las representaciones geométricas, verbales y analíticas. Además, expresaron en lenguaje analítico la acumulación de diferencias mediante las fórmulas de las sumas inferiores y superiores, en lugar de usar una más sencilla sugerida por el rectángulo de acumulación de diferencias que aparece en el applet.

Los estudiantes del perfil 3 fueron capaces de expresar de forma correcta verbal y analíticamente la base y la altura de los rectángulos que recubren la superficie, tanto para las sumas inferiores como para las superiores coordinando las representaciones geométricas, verbales y analíticas, aunque las dejaron expresadas en términos de $f\left(x_{i}\right)$ sin sustituir en la función $\left(x_{i}^{2}\right)$. Sin embargo, no fueron capaces de expresar verbalmente la sumas inferiores y superiores. Además, manifestaron que el extremo del subintervalo donde tenían que calcular el valor de la función para identificar la altura del rectángulo 
dependía de que la función fuera creciente o decreciente en dicho intervalo. Por otra parte, relacionaron el incremento del número de subintervalos con una cota del error de la aproximación y con el recubrimiento de la superficie por rectángulos. También relacionan las sumas superiores/inferiores con la suma de las áreas de los rectángulos. Infieren que a mayor número de subintervalos mejor es la aproximación del área bajo la curva y que el error es una cota y coordinan los distintos sistemas de representación.

\section{Conclusiones}

En esta sección presentamos en primer lugar nuestras conclusiones en relación con el avance de los estudiantes en la construcción del conocimiento con ayuda de estas tareas y en segundo lugar algunas implicaciones para la enseñanza que consideramos relevantes.

\section{Contexto de construcción del conocimiento}

En este trabajo hemos introducido el concepto de integral definida como límite para calcular el área bajo la curva. Los estudiantes habían trabajado previamente el concepto de límite, pero la forma en que abordaron la tarea "Área bajo un cuadrante" nos mostró que este no fue un ejercicio de aplicación de algo que ya sabían, sino que les ayudó a avanzar el concepto de aproximación. Algunos de ellos construyeron las concepciones dinámica y métrica del límite, y otros consiguieron coordinar ambas.

Este avance en el conocimiento pensamos que fue favorecido por el trabajo realizado por los estudiantes a partir de las tareas presentadas. Se les pedía que experimentaran y reflexionaran sobre los resultados de su experimentación, al tiempo que trabajaban con distintos sistemas de representación y además de forma simultánea e interactiva. Por otra parte, el trabajo se realizó por parejas y un trío, y esta interacción verbal pudo favorecer el avance en la comprensión conceptual. Otras investigaciones lo confirman (Aranda \& Callejo, 2010a; Berry \& Nyman, 2003; Camacho, Santos \& Depool, 2013). Por otra parte, Camacho et al., (2013) señalan que es importante la discusión entre estudiantes de las distintas representaciones para facilitar la comprensión de la integral.

En cuanto al papel de los applets, Ferrara el al. (2006) sugerían que relacionar distintas representaciones en la pantalla podía facilitar que se construyeran las conexiones necesarias. En esta investigación se confirma que usar simultáneamente distintas representaciones dinámicas e interactivas puede ayudar en la construcción del concepto, y 
ayuda a los estudiantes a superar la visión algorítmica del Cálculo, permitiéndoles desarrollar una mejor comprensión de la aproximación gráfica al Cálculo (Aranda \& Callejo, 2017b).

\section{Implicaciones para la enseñanza}

En este entorno de aprendizaje, cambia el papel de los estudiantes y del profesor. Los estudiantes son protagonistas de su aprendizaje, perciben las matemáticas como una ciencia experimental y pueden desarrollar sus competencias. El profesor adapta el diseño original al ritmo de aprendizaje de los estudiantes, orientándolos, favoreciendo su autonomía y objetivando el conocimiento

El trabajo en grupo, al verbalizar el pensamiento de los estudiantes, discutiendo sus ideas, permite al profesor observar mejor el proceso de construcción del conocimiento. Por otra parte, cada grupo puede avanzar a su ritmo, y los más avanzados pueden compartir sus conocimientos con el resto en las puestas en común

Consideramos que hay que destacar algunos aspectos que favorecen un trabajo en grupo eficaz son el disponer de una guía de trabajo proponiendo acciones como experimentar, conjeturar, comprobar, justificar o elaborar conclusiones y que los grupos sean de nivel académico similar.

El diseño de esta unidad didáctica usó la tecnología para ahorrar cálculos tediosos a los estudiantes, pero sobre todo para facilitar la experimentación y el trabajo simultáneo con distintos sistemas de representación. Las tecnologías son también instrumentos de mediación semiótica y permiten a los estudiantes identificar, examinar, conectar y relacionar las diferentes representaciones del concepto (Camacho, Santos y Depool, 2013).

\section{Referencias}

Apostol, T. M. (1960). Análisis matemático. Reverté.

Aranda, C. (2015). Análisis de la construcción del concepto de integral definida en estudiantes de bachillerato. Tesis doctoral. Universidad de Alicante. http://hdl.handle.net/10045/53161

Aranda, C. \& Callejo, M.L. (2010a). Construcción del concepto de dependencia lineal en un contexto de geometría dinámica: Un estudio de casos. Revista Latinoamericana de Investigación en Matemática Educativa, 13(2), 129-158. http://www.scielo.org.mx/pdf/relime/v13n2/v13n2a2.pdf 
Aranda, C. \& Callejo, M. L. (2010b). Un experimento de enseñanza para la construcción del concepto de integral definida usando un programa de geometría dinámica. En: J. L. Galán, G. Aguilera y P. Rodríguez (Eds.), Proceedings of TIME 2010. Universidad de Málaga.

Aranda, C. \& Callejo, M. L., (2017a) Formas de aproximar el área bajo una curva: un estudio con estudiantes de bachillerato. Enseñanza de las Ciencias, 35(1), pp. 157174. https://doi.org/10.5565/rev/ensciencias. 2075

Aranda, C. \& Callejo, M. L. (2017b). Construcción de la función integral y razonamiento covariacional: dos estudios de casos. Bolema: Boletim de Educação Matemática, 31(58), 777-798. https://doi.org/10.1590/1980-4415v31n58a13.

Artigue, M. (1991). Analysis. In D. Tall (Ed.), Advanced Mathematical Thinking (pp. 167198). Kluwer Academic Publishers.

Azcárate, C., Casadevall, M., Casellas, E. \& Bosch, D. (1996). Cálculo diferencial e integral. Síntesis.

Berry, J. S. \& Nyman, M. A. (2003). Promoting students' graphical understanding of the calculus. Journal of Mathematical Behavior, 22, 481-497.

Blázquez, S. y Ortega, T. (2002). Nueva definición de límite funcional. UNO, Revista de Didáctica de las Matemáticas 30, 67-83.

Blume, G. W. \& Heid, M. K. (2008). The role of research theory in the integration of technology in mathematics teaching and learning. In: M. K. Heid \& G. W. Blume (Eds.), Research on Technology and the Teaching and Learning of Mathematics. Research Syntheses (Vol. 2, pp. 449-464). Charlotte, North Carolina: National Council of Teachers of Mathematics-IAP.

Boigues, F. J., Llinares, S. \& Estruch, V. D. (2010). Desarrollo de un esquema de integral definida en estudiantes de ingenierías relacionadas con las ciencias de la naturaleza. RELIME, 13(3), pp.129-158. http://relime.org/index.php/numeros/todosnumeros/volumen-13/numero-13-3/524-201001c

Botella, L. M., Cascón, B., Martín, C., Millán, L. M., Moltó, C., Pérez, C. \& Salinas, E (2001). Matemàtiques 2. Marfil.

Camacho, M., Santos, M. \& Depool, R. (2013) La resolución de problemas, tecnología y comprensión del concepto de integral definida. Una investigación con estudiantes de ingeniería. UNO, Revista de Didáctica de las Matemáticas, 63, 50-68.

Codes, M., Sierra, M. \& Raboso, M. (2007). Innovación en la recogida de datos para una investigación de carácter cualitativo. Un ejemplo con alumnos universitarios en un entorno computacional. En: M. Camacho, P. Flores y P. Bolea (Eds.), Investigación en Educación Matemática XI (pp. 261-271). SEIEM.

Cornu, B. (1991). Limits. In: D. Tall (Ed), Advanced Mathematical Thinking (pp. 153 166). Kluver.

Courant, R. \& Robbins, H. (2002). ¿Qué son las matemáticas? Fondo de Cultura Económica.

Dreyfus, T. (1991). Advanced mathematical thinking processes. In: D. Tall (Ed.), Advanced Mathematical Thinking (pp. 25-41). Kluwer Academic Publisher. 
Dreyfus, T. \& Eisenberg, T. (1986). On visual versus analytical thinking in mathematics. Proceedings of the 10th conference of the International Group for the Psychology of Mathematics Education (pp. 153-158). University of London, Institute of Education.

Duval, R. (1993). Registres de représentation sémiotique et fonctionnement cognitif de la pensée. Annales de Didactique et de Sciences Cognitives, 5, pp. 37- 65.

Duval, R. (2006). Un tema crucial en la educación matemática: la habilidad para cambiar el registro de representación. La Gaceta de la Real Sociedad Matemática Española, 9 (1), 143-168.

Ferrara, F., Pratt, D. \& Robutti, O. (2006). The role and uses of technologies for the teaching of algebra and calculus. In: A. Gutierrez \& P. Boero (Eds.), Handbook of Research on the Psychology of Mathematics Education. Past, Present and Future (pp. 237-274). Sense Publishers.

Ferrini-Mundy, J. \& Graham, K. (1994). Research in Calculus learning: understanding of limits, derivatives, and integrals. In: J. Kaput \& E. Dubinsky (Eds.), Research issues in undergraduate Mathematics Learning: Preliminary Analyses and Results, MAA Notes Number 33 (pp. 31-45). Mathematical Association of America.

González-Martín, A.S. \& Camacho, M, (2004). What is first-year mathematics student's actual knowledge about improper integrals? International Journal of Mathematical Education in Science and Technology, 35(1), 73-89.

Guzmán, M. (2010). El rincón de la pizarra. Pirámide.

Ferrara, F., Pratt, D. \& Robutti, O. (2006). The role and uses of technologies for the teaching of algebra and calculus. In: A. Gutierrez \& P. Boero (Eds.), Handbook of Research on the Psychology of Mathematics Education. Past, Present and Future (pp. 237-274). Sense Publishers.

Heid, M. K. \& Blume, G. W. (2008). Algebra and function development. In: M. K. Heid \& G. W. Blume (Eds.), Research on Technology and the Teaching and Learning of Mathematics. Research Syntheses (vol. 1, pp. 55-108). National Council of Teachers of Mathematics-IAP.

Hong, Y. \& Thomas, M. (1997). Using the computer to improve conceptual thinking in integration. In: E. Pehkonen (Ed.), Proceedings of the 21st Conference of the International Group for the Psychology of Mathematics Education (vol. 3, pp. 81-88). University of Helsinki.

Kieran, C. \& Drijvers, P. (2006). The co-emergence of machine techniques, paper-andpencil techniques, and theoretical reflection: a study of CAS use in secondary school algebra. International Journal of Computers for Mathematical Learning, 11, 205-263.

Lagrange, J. B. \& Artigue, M. (2009). Student's activities about functions at upper secondary level: A grid for designing a digital environment and analyzing uses. In: M. Tzekaki, M. Kaldrimidou y H. Sakonidis (Eds.), Proceedings of the $33^{\text {rd }}$ Conference of the International Group for the Psychology of Mathematics Education (vol. 3, pp. 465-472). PME.

Maschietto, M. (2008). Graphic calculators and micro-straightness: analysis of a didactic engineering. International Journal of Computers for Mathematical Learning, 13(3), 207-230.

Pons, J. B. (2014). Análisis de la comprensión en Estudiantes de bachillerato del concepto de límite de una función en un punto. Tesis doctoral. Universidad de Alicante. 
Pons, J., Valls, J. \& Llinares, S. (2011). Coordination of aproximation in secondary school students' understanding of limit concept. In: B. Ubuz (Ed.), Proceedings of the $35^{\text {th }}$ Conference of the International Group for the Psychoogy of Mathematics Education (vol. 3, pp. 393-400). PME.

Porres, M. (2012). Integral definida, cálculo mental y nuevas tecnologías. Tesis doctoral. Universidad de Valladolid. http://uvadoc.uva.es/handle/10324/949

Porres, M., Pecharromán, C. \& Ortega, T. (2017). Aportaciones de DERIVE y del cálculo mental al aprendizaje de la integral definida. PNA: Revista de Didáctica de la Matemática, 11(2), 125-153. https://revistaseug.ugr.es/index.php/pna/article /view/6077

Orton, A. (1983). Students' understanding of integration. Educational Studies in Mathematics, 14(1), 1-18.

Sánchez-Matamoros, G., García, M. \& Llinares, S. (2006). El desarrollo del esquema de derivada. Enseñanza de las Ciencias, 24(1), 85-89.

Sealey, V. (2006). Student understanding of definite integrals, Riemann sums and area under a curve: What is necessary and sufficient? In: S. Alatorre, J. L. Cortina, M. Sáiz, \& A. Méndez (Eds.), Proceedings of the 28th annual meeting of the North American Chapter of the International Group for the Psychology of Mathematics Education (vol. 2, pp. 46-53). Universidad Pedagógica Nacional.

Sealey, V. (2014). A framework for characterizing student understanding of Riemann sums and definite integrals. The Journal of Mathematical Behavior, 33, 230-245.

Swidan, O. \& Yerushalmy, M. (2014). Learning the indefinite integral in a dynamic and interactive technological environment. ZDM, 46(4), 517-531.

Tall, D., Smith, D. \& Piez, C. (2008). Technology and calculus. In: M. K. Heid \& G. W. Blume (Eds.), Research on Technology and Learning of Mathematics. Research Syntheses (vol. 1, pp. 207-258). NCTM.

Turégano, P. (1998). Del área a la integral. Un estudio en el contexto educativo. Enseñanza de las Ciencias, 16(2), 233-249.

Valls, J., Pons, J., \& Llinares, S. (2011). Coordinación de los procesos de aproximación en la comprensión del límite de una función. Enseñanza de las ciencias, 29(3), 325-338.

Autoras

María Carmen Aranda López

Doctora en Didáctica de la Matemática por la Universidad de Alicante (UA). Premio extraordinario de Doctorado por la misma Universidad. Profesora de Matemáticas de Secundaria del Instituto de Educación Secundaria (I.E.S), Alicante, España. E-mail:

carmen.arlo@gmail.com

María Luz Callejo de La Vega

Doctora en Didáctica de las Disciplinas, opción Matemáticas, por la Universidad Denis Diderot (Paris 7). Profesora Titular de Didáctica de la Matemática del Departamento de Innovación y Formación Didáctica de la Universidad de Alicante, España. E-mail: 